\title{
Relating Gauge and Gravity Theories in the Large Mass Limit
}

\author{
Kays Haddad $\odot^{*}$ and Andreas Helset $\odot^{\dagger}$ \\ Niels Bohr International Academy \& Discovery Center, Niels Bohr Institute, University of Copenhagen, \\ Blegdamsvej 17, DK-2100 Copenhagen, Denmark
}

(Received 6 June 2020; revised 7 August 2020; accepted 10 September 2020; published 30 October 2020)

\begin{abstract}
We show how to double copy heavy quark effective theory (HQET) to heavy black hole effective theory (HBET) for spin $s \leq 1$. In particular, the double copy of spin-s HQET with scalar QCD produces spin- $s$ HBET, while the double copy of spin-1/2 HQET with itself gives spin-1 HBET. Finally, we present novel all-order-in-mass Lagrangians for spin-1 heavy particles.
\end{abstract}

DOI: 10.1103/PhysRevLett.125.181603

Introduction.-An expanding family of field theories has been observed to obey double copy relations [1-36] (for a review of the double-copy program, see Ref. [37]). In particular, scattering amplitudes of gravitational theories with massive matter can be calculated from the double copy of gauge theories with massive matter [38-51].

As heavy quark effective theory (HQET) [52] is derived from QCD and heavy black hole effective theory (HBET) [53] is derived from gravity with massive particles, HBET amplitudes should be obtainable as double copies of HQET amplitudes. Indeed, this is the main result of this Letter. We show through direct computation that the tree-level threepoint and Compton amplitudes of HQET and HBET satisfy the schematic relations

$$
\begin{gathered}
\left(\mathrm{QCD}_{s=0}\right) \times\left(\mathrm{HQET}_{s}\right)=\mathrm{HBET}_{s}, \\
\left(\mathrm{HQET}_{s=1 / 2}\right) \times\left(\mathrm{HQET}_{s=1 / 2}\right)=\mathrm{HBET}_{s=1},
\end{gathered}
$$

for $s \leq 1$, where the spin- $s$ HQET and HBET matter states are equal in the free-field limit, and the spin-1 heavy polarization vectors are related to the heavy spinors through Eq. (29). While we only show here the double copy for three-point and Compton amplitudes, invariance of the $S$ matrix under field redefinitions implies that Eq. (1) holds more generally whenever QCD double copies to gravitationally interacting matter. Equation (1) expands the double copy in powers of $\hbar$ since the operator expansion for heavy particles can be interpreted as an expansion in $\hbar$ [53]. The $\hbar \rightarrow 0$ limit of the double copy is currently of particular relevance for gravitational wave physics $[41,45,46]$.

Published by the American Physical Society under the terms of the Creative Commons Attribution 4.0 International license. Further distribution of this work must maintain attribution to the author(s) and the published article's title, journal citation, and DOI. Funded by SCOAP ${ }^{3}$.
We will begin in the second section with a brief review of the color-kinematics duality, and we will also discuss double copying with effective matter fields. In the next two sections we demonstrate the double copy at tree level for three-point and Compton amplitudes for spins $0,1 / 2$, and 1 , respectively. We offer our conclusions in the final section. The Lagrangians used to produce the amplitudes in this Letter are presented in the Supplemental Material [54]. Among them are novel all-order-in-mass Lagrangians for spin-1 HQET and HBET.

Color-kinematics duality and heavy fields.-A tree-level $n$-point gauge-theory amplitude, potentially with external matter, can be written as follows (we omit coupling constants for the sake of clarity; reinstating them is straightforward: after double copying the gauge theory coupling undergoes the replacement $g_{s} \rightarrow \sqrt{\kappa} / 2$ ):

$$
\mathcal{A}_{n}=\sum_{i \in \Gamma} \frac{c_{i} n_{i}}{d_{i}}
$$

where $\Gamma$ is the set of all diagrams with only cubic vertices. Also, $c_{i}$ are color factors, $n_{i}$ encode the kinematic information, and $d_{i}$ are propagator denominators. A subset of the color factors satisfies the identity

$$
c_{i}+c_{j}+c_{k}=0 .
$$

If the corresponding kinematic factors satisfy the analogous identity,

$$
n_{i}+n_{j}+n_{k}=0,
$$

and have the same antisymmetry properties as the color factors, then the color and kinematic factors are dual. In this case, the color factors in Eq. (2) can be replaced by kinematic factors to form the amplitude

$$
\mathcal{M}_{n}=\sum_{i \in \Gamma} \frac{n_{i}^{\prime} n_{i}}{d_{i}}
$$


which is a gravity amplitude with antisymmetric tensor and dilaton contamination. [For an amplitude of arbitrary multiplicity containing massive external states with an arbitrary spectrum, Eq. (5) may not represent a physical amplitude [56]. However, for the cases under consideration in this Letter, the application of the double copy will yield a well-defined gravitational amplitude.] In general, $n_{i}^{\prime}$ and $n_{i}$ need not come from the same gauge theory, and only one of the sets must satisfy the color-kinematics duality.

In this Letter, we are interested in applying the doublecopy procedure to HQET. A complicating factor to double copying effective field theories (EFTs) is that Lagrangian descriptions of EFTs are not unique, as the Lagrangian can be altered by redefining one or more of the fields. The Lehmann, Symanzik, and Zimmermann (LSZ) procedure [57] guarantees the invariance of the $S$ matrix, and in particular Eqs. (2) and (5), under such field redefinitions by accounting for wave function normalization factors (WNFs) $\mathcal{R}^{-1 / 2}$, which contribute to the on-shell residues of two-point functions. Note that $\mathcal{R}^{-1 / 2}=1$ for canonically normalized fields. The WNF for an effective state $\tilde{\varepsilon}$ can thus be determined by relating it to a canonically normalized state $\varepsilon$ through

$$
\varepsilon=\mathcal{R}^{-1 / 2} \cdot \tilde{\varepsilon}
$$

Under the double copy the WNFs from each matter copy combine in a spin-dependent manner, which complicates the matching of the double-copied amplitude to one derived from a gravitational Lagrangian.

In order to ease the double copying of HQET to HBET, we would like to avoid having to compensate for the WNFs. This can be achieved by ensuring that HQET and HBET have the same WNFs-i.e., that the asymptotic states for the spin-s particles in HQET and HBET are equal - and double copying HQET with QCD, which has a trivial WNF.

The asymptotic states - that is, the states in the free-field limit — of the canonically normalized theories (given by complex Klein-Gordon, Dirac, and symmetry-broken Proca actions) are related to their respective asymptotic heavy states (labeled by a velocity $v$ ) in position space through

$$
\begin{aligned}
& \varphi(x)=\frac{e^{-i m v x}}{\sqrt{2 m}}\left[1-\frac{1}{2 m+i v \cdot \partial+\frac{\partial_{\perp}^{2}}{2 m}} \frac{\partial_{\perp}^{2}}{2 m}\right] \phi_{v}(x), \\
& \psi(x)=e^{-i m v x}\left[1+\frac{i}{2 m+i v \cdot \partial}(\not \partial-v \partial)\right] Q_{v}(x), \\
& A^{\mu}(x)=\frac{e^{-i m v x}}{\sqrt{2 m}}\left[\delta_{\nu}^{\mu}-\frac{i v^{\mu} \partial_{\nu}-\partial^{\mu} \partial_{\nu} / 2 m}{m+i v \cdot \partial / 2}\right] B_{v}^{\nu}(x),
\end{aligned}
$$

where $a_{\perp}^{\mu}=a^{\mu}-v^{\mu}(v a)$ for a vector $a^{\mu}$. Here, the momentum is decomposed as $p^{\mu}=m v^{\mu}+k^{\mu}$ in the usual heavy- particle fashion. The Lagrangians for the heavy fields in Eq. (7) are given in the Supplemental Material [54]. Converting to momentum space, Eq. (7) gives the WNFs

$$
\begin{aligned}
& \mathcal{R}_{s=0}^{-1 / 2}(p)=\frac{1}{\sqrt{2 m}}\left[1+\frac{k_{\perp}^{2}}{4 m^{2}+2 m v \cdot k-k_{\perp}^{2}}\right], \\
& \mathcal{R}_{s=1 / 2}^{-1 / 2}(p)=1+\frac{1}{2 m+v \cdot k}(\not k-v \cdot k), \\
& \left(\mathcal{R}_{s=1}^{-1 / 2}(p)\right)_{\mu}^{\nu}=\frac{1}{\sqrt{2 m}}\left[\delta_{\mu}^{\nu}-\frac{v_{\mu} k^{\nu}+k_{\mu} k^{\nu} / 2 m}{m+v \cdot k / 2}\right] .
\end{aligned}
$$

We will demonstrate that spin-s HBET amplitudes can directly be obtained by double copying spin- $s$ HQET amplitudes with scalar QCD for spins $s \leq 1$. At $s=1$ there is also the possibility to double copy using two spin$1 / 2$ amplitudes. We will discuss this point further below.

Spin-O gravitational amplitudes.-We begin with the simplest case of spinless amplitudes. Consider first the three-point amplitude. For scalar HQET we have that [with $\left.\phi_{v}^{*}=\phi_{v}^{*}\left(p_{2}\right), \phi_{v}=\phi_{v}\left(p_{1}\right)\right]$

$$
\begin{aligned}
\mathcal{A}_{3}^{H, s=0}= & -\mathbf{T}_{i j}^{a} \epsilon_{q}^{* \mu} \phi_{v}^{*}\left(1+\frac{k_{1}^{2}+k_{2}^{2}}{4 m^{2}}\right) \phi_{v} \\
& \times\left[v_{\mu}+\frac{\left(k_{1}+k_{2}\right)_{\mu}}{2 m}\right]+\mathcal{O}\left(m^{-4}\right),
\end{aligned}
$$

where $k_{2}=k_{1}-q$. For scalar QCD the amplitude is

$$
\mathcal{A}_{3}^{s=0}=-\mathbf{T}_{i j}^{a} \epsilon_{q}^{* \mu}\left[2 m v_{\mu}+\left(k_{1}+k_{2}\right)_{\mu}\right] .
$$

Note that we have left the external heavy scalar factor $\phi_{v}$ explicit in the HQET amplitude. This is because, in contrast to the canonically normalized scalar fields, the heavy scalar factors are not equal to 1 in momentum space. Indeed, for the HQET amplitude to be equal to the QCD amplitude, the heavy scalar factor in momentum space must be equal to the inverse of Eq. (8a). This will cancel the extra factor in round brackets in Eq. (9) up to $\mathcal{O}\left(m^{-4}\right)$. Note that $k_{\perp}^{2}=k^{2}+\mathcal{O}\left(m^{-2}\right)$.

The double copy at three points is simply given by a product of amplitudes:

$$
\begin{aligned}
\mathcal{A}_{3}^{s=0} & \mathcal{A}_{3}^{H, s=0}=\epsilon_{q}^{* \mu} \epsilon_{q}^{* \nu} \phi_{v}^{*}\left(1+\frac{k_{1}^{2}+k_{2}^{2}}{4 m^{2}}\right) \phi_{v} \\
& \times 2 m\left[v_{\mu} v_{\nu}+v_{\mu} \frac{k_{1 \nu}+k_{2 \nu}}{m}+\frac{\left(k_{1}+k_{2}\right)_{\mu}\left(k_{1}+k_{2}\right)_{\nu}}{4 m^{2}}\right] \\
& +\mathcal{O}\left(m^{-3}\right) .
\end{aligned}
$$

As the only massless particle in this process is external, we can easily eliminate the massless nongraviton degrees of freedom by identifying the outer product of gluon 
polarization vectors with the graviton polarization tensor. After doing so, Eq. (11) agrees with the three-point amplitude derived from the Supplemental Material [54].

As another example, consider the Compton amplitude. The color decomposition for Compton scattering is

$$
\mathcal{A}_{4}^{s}=\frac{c_{s} n_{s}}{d_{s}}+\frac{c_{t} n_{t}}{d_{t}}+\frac{c_{u} n_{u}}{d_{u}},
$$

where

$c_{s}=\mathbf{T}_{i k}^{a} \mathbf{T}_{k j}^{b}, \quad c_{t}=i f^{a b c} \mathbf{T}_{i j}^{c}, \quad c_{u}=\mathbf{T}_{i k}^{b} \mathbf{T}_{k j}^{a}$.

[We have computed all Compton amplitudes using NRQCD propagators. It is also possible to perform the computations using HQET propagators: in that case, a comparison to the Compton amplitude for the emission of biadjoint scalars from heavy particles (described by the Lagrangians in the Supplemental Material [54]) — analogous to the treatment in Ref. [58] - is necessary to identify kinematic numerators. Both methods produce the same results.] The kinematic numerators for scalar HQET are

$$
\begin{gathered}
n_{s}^{H, s=0}=-2 m \phi_{v}^{*} \epsilon_{q_{1}}^{* u} \epsilon_{q_{2}}^{* \nu} v_{\mu} v_{\nu}\left(1+\frac{k_{1}^{2}+k_{2}^{2}}{4 m^{2}}\right) \phi_{v}, \\
n_{t}^{H, s=0}=0, \\
n_{u}^{H, s=0}=\left.n_{s}^{H, s=0}\right|_{q_{1} \leftrightarrow q_{2}},
\end{gathered}
$$

where $k_{2}=k_{1}-q_{1}-q_{2}$. Those for scalar QCD are

$$
\begin{aligned}
& n_{s}^{s=0}=-4 m^{2} \epsilon_{q_{1}}^{* \mu} \epsilon_{q_{2}}^{* \nu} v_{\mu} v_{\nu}, \\
& n_{t}^{s=0}=0, \\
& n_{u}^{s=0}=\left.n_{s}^{s=0}\right|_{q_{1} \leftrightarrow q_{2}} .
\end{aligned}
$$

For brevity we have written the numerators under the conditions $k_{1}=q_{i} \cdot \epsilon_{j}=\epsilon_{i} \cdot \epsilon_{j}=0$; the initial residual momentum can always be set to 0 by reparametrizing $v$, and such a gauge exists for opposite helicity gluons. We have checked explicitly up to and including $\mathcal{O}\left(\mathrm{m}^{-2}\right)$ that the following results hold when relaxing all of these conditions.

Both the HQET and QCD numerators satisfy the colorkinematics duality in the form

$$
c_{s}-c_{u}=c_{t} \Leftrightarrow n_{s}-n_{u}=n_{t} .
$$

We can therefore replace the color factors in the HQET amplitude with the QCD kinematic numerators,

$$
\mathcal{M}_{4}^{H, s=0}=\frac{n_{s}^{s=0} n_{s}^{H, s=0}}{d_{s}}+\frac{n_{t}^{s=0} n_{t}^{H, s=0}}{d_{t}}+\frac{n_{u}^{s=0} n_{u}^{H, s=0}}{d_{u}} .
$$

Identifying once again the outer products of gluon polarization vectors with graviton polarization tensors, we find that the Compton amplitude derived from the Supplemental Material [54] agrees with Eq. (16).

To summarize, we have explicitly verified that

$$
\left(\mathrm{QCD}_{s=0}\right) \times\left(\mathrm{HQET}_{s=0}\right)=\mathrm{HBET}_{s=0}
$$

for three-point and Compton amplitudes.

Spin-1/2 gravitational amplitudes.-We now move on to the double copy of spin-1/2 HQET with scalar QCD to obtain spin-1/2 HBET. The three-point spin-1/2 HQET amplitude is [with $\bar{u}_{v}=\bar{u}_{v}\left(p_{2}\right), u_{v}=u_{v}\left(p_{1}\right)$ ]

$$
\begin{aligned}
\mathcal{A}_{3}^{H, s=1 / 2}= & -\mathbf{T}_{i j}^{a} \bar{u}_{v} u_{v} \epsilon_{q}^{* \mu}\left(v_{\mu}+\frac{k_{1 \mu}}{m}+\frac{k_{1}^{2}-k_{1} \cdot q}{4 m^{2}} v_{\mu}\right) \\
& -\frac{i \mathbf{T}_{i j}^{a}}{2 m} \bar{u}_{v} \sigma^{\alpha \beta} u_{v} \epsilon_{q}^{* \mu}\left[q_{\alpha} \eta_{\beta \mu}-\frac{1}{2 m} q_{\alpha} k_{1 \beta} v_{\mu}\right] \\
& +\mathcal{O}\left(m^{-3}\right) .
\end{aligned}
$$

Double copying with scalar QCD, we find

$$
\mathcal{M}_{3}^{H, s=1 / 2}=\mathcal{A}_{3}^{s=0} \mathcal{A}_{3}^{H, s=1 / 2},
$$

where $\mathcal{M}_{3}^{H, s=1 / 2}$ is the amplitude derived from the Supplemental Material [54].

We turn now to Compton scattering. For brevity we write here the amplitudes in the case $k_{1}=q_{i} \cdot \epsilon_{j}=\epsilon_{i} \cdot \epsilon_{j}=0$. We have checked explicitly that the results hold when these conditions are relaxed. Also, we have performed the calculation up to $\mathcal{O}\left(\mathrm{m}^{-2}\right)$ but only present the kinematic numerators up to $\mathcal{O}\left(\mathrm{m}^{-1}\right)$. They are

$$
\begin{gathered}
n_{s}^{H, s=1 / 2}=-2 m \bar{u}_{v}\left[v \cdot \epsilon_{q_{1}}^{*} v \cdot \epsilon_{q_{2}}^{*}\right. \\
\left.-\frac{i v_{\rho}}{2 m} \sigma_{\mu \nu}\left(\epsilon_{q_{1}}^{* \mu} q_{1}^{\nu} \epsilon_{q_{2}}^{* \rho}+\epsilon_{q_{2}}^{* \mu} q_{2}^{\nu} \epsilon_{q_{1}}^{* \rho}-q_{2}^{\rho} \epsilon_{q_{2}}^{* \mu} \epsilon_{q_{1}}^{* \nu}\right)\right] u_{v}, \\
n_{t}^{H, s=1 / 2}=0 \\
n_{u}^{H, s=1 / 2}=\left.n_{s}^{H, s=1 / 2}\right|_{q_{1} \leftrightarrow q_{2}} .
\end{gathered}
$$

In this case, the color-kinematic duality Eq. (15) is violated at $\mathcal{O}\left(\mathrm{m}^{-2}\right)$. Nevertheless, since the scalar QCD kinematic numerators satisfy the duality we can use them to double copy the spin-1/2 Compton amplitude. Doing so we find

$$
\mathcal{M}_{4}^{H, s=1 / 2}=\frac{n_{s}^{s=0} n_{s}^{H, s=1 / 2}}{d_{s}}+\frac{n_{t}^{s=0} n_{t}^{H, s=1 / 2}}{d_{t}}+\frac{n_{u}^{s=0} n_{u}^{H, s=1 / 2}}{d_{u}},
$$


where $\mathcal{M}_{4}^{H, s=1 / 2}$ is the spin-1/2 HBET Compton amplitude derived from the Supplemental Material [54].

We have seen that

$$
\left(\mathrm{QCD}_{s=0}\right) \times\left(\mathrm{HQET}_{s=1 / 2}\right)=\mathrm{HBET}_{s=1 / 2}
$$

for the three-point and Compton amplitudes.

Spin-1 gravitational amplitudes.-Gravitational amplitudes with spin-1 matter can be obtained by double copying two gauge theories with matter in two ways: spin- $0 \times$ spin1 or spin- $1 / 2 \times$ spin- $1 / 2[47,49,50]$. This fact also holds for heavy particles. We now show this in two examples by deriving the spin-1 gravitational three-point and Compton amplitudes using both double-copy procedures.

Double copy: The three-point spin-1 HQET amplitude is

$$
\begin{aligned}
\mathcal{A}_{3}^{H, s=1}= & \mathbf{T}_{i j}^{a} \varepsilon_{v}^{* \beta} \varepsilon_{v}^{\alpha} \epsilon_{q}^{* \mu}\left[\eta_{\alpha \beta} v_{\mu}\right. \\
& +\frac{1}{2 m}\left[\eta_{\alpha \beta}\left(k_{1}+k_{2}\right)_{\mu}-2 q_{\beta} \eta_{\alpha \mu}+2 q_{\alpha} \eta_{\beta \mu}\right] \\
& \left.+\frac{1}{2 m^{2}} v_{\mu}\left(-k_{1 \beta} q_{\alpha}+q_{\alpha} q_{\beta}+q_{\beta} k_{1 \alpha}\right)\right],
\end{aligned}
$$

where $k_{2}^{\mu}=k_{1}^{\mu}-q^{\mu} \quad$ and $\quad \varepsilon_{v}^{* \mu}=\varepsilon_{v}^{* \mu}\left(p_{2}\right), \varepsilon_{v}^{\mu}=\varepsilon_{v}^{\mu}\left(p_{1}\right)$. Double copying with scalar QCD we find

$$
\mathcal{M}_{3}^{H, s=1}=\mathcal{A}_{3}^{s=0} \mathcal{A}_{3}^{H, s=1},
$$

where $\mathcal{M}_{3}^{H, s=1}$ is the amplitude derived from the Supplemental Material [54].

Compton scattering for spin-1 HQET is given by the kinematic numerators

$$
\begin{gathered}
n_{s}^{H, s=1}=2 m \varepsilon_{v}^{* \beta} \varepsilon_{v}^{\alpha}\left[v \cdot \epsilon_{q_{1}}^{*} v \cdot \epsilon_{q_{2}}^{*} \eta_{\alpha \beta}\right. \\
+\frac{v_{\rho}}{m}\left(\eta_{\alpha \nu} \eta_{\beta \mu}-\eta_{\alpha \mu} \eta_{\beta \nu}\right)\left(\epsilon_{q_{1}}^{* \mu} q_{1}^{\nu} \epsilon_{q_{2}}^{* \rho}+\epsilon_{2}^{* \mu} q_{2}^{\nu} \epsilon_{q_{1}}^{* \rho}\right) \\
\left.-\frac{v q_{2}}{2 m}\left(\epsilon_{q_{1} \alpha}^{*} \epsilon_{q_{2} \beta}^{*}-\epsilon_{q_{2} \alpha}^{*} \epsilon_{q_{1} \beta}^{*}\right)\right], \\
n_{t}^{H, s=1}=0, \\
n_{u}^{H, s=1}=\left.n_{s}^{H, s=1}\right|_{q_{1} \leftrightarrow q_{2}},
\end{gathered}
$$

where, for brevity, we again write the numerators up to $\mathcal{O}\left(m^{-1}\right)$ and in the case where $k_{1}=\epsilon_{i} \cdot \epsilon_{j}=q_{i} \cdot \epsilon_{j}=0$. We have performed the calculation up to $\mathcal{O}\left(m^{-2}\right)$ and checked the general case explicitly. The double copy becomes

$\mathcal{M}_{4}^{H, s=1}=\frac{n_{s}^{s=0} n_{s}^{H, s=1}}{d_{s}}+\frac{n_{t}^{s=0} n_{t}^{H, s=1}}{d_{t}}+\frac{n_{u}^{s=0} n_{u}^{H, s=1}}{d_{u}}$,

where $\mathcal{M}_{4}^{H, s=1}$ is derived from the Supplemental Material [54].
Thus, we find that

$$
\left(\mathrm{QCD}_{s=0}\right) \times\left(\mathrm{HQET}_{s=1}\right)=\mathrm{HBET}_{s=1}
$$

for three-point and Compton amplitudes.

$1 / 2 \times 1 / 2$ double copy: The spin-1 gravitational amplitudes can also be obtained by double copying the spin- $1 / 2$ HQET amplitudes. To do so, we use the on-shell heavy particle effective theory (HPET) variables of Ref. [59] to modify Eq. (2.11) of Ref. [50] for the case of heavy particles. Using the fact that the on-shell HPET variables correspond to momenta $p_{v}^{\mu}=m_{k} v^{\mu}$ with mass $m_{k}=m\left(1-k^{2} / 4 m^{2}\right)$, following the derivation of Ref. [50] leads to

$$
\mathcal{M}_{n}^{H, 1 / 2 \times 1 / 2}=\frac{m_{k_{1}} m_{k_{2}}}{m} \sum_{\alpha \beta} K_{\alpha \beta} \operatorname{Tr}\left[\mathcal{A}_{n, \alpha}^{H, 1 / 2} P_{+} \phi_{v} \overline{\mathcal{A}}_{n, \beta}^{H, 1 / 2} P_{-} \phi_{v}^{*}\right],
$$

where $P_{ \pm}=(1 \pm \not p) / 2, K_{\alpha \beta}$ is the KLT kernel, and $\alpha, \beta$ represent color orderings. Here $\mathcal{A}^{H}$ and $\overline{\mathcal{A}}^{H}$ are amplitudes with the external states stripped, and $\overline{\mathcal{A}}^{H}=-\gamma_{5}\left(\mathcal{A}^{H}\right)^{\dagger} \gamma_{5}$. We have also adopted the convention that only the initial matter momentum is incoming. Converting to the on-shell HPET variables, it can be easily seen that

$$
\varepsilon_{v \mu}^{I J}(p)=\frac{1}{2 \sqrt{2} m_{k}} \bar{u}_{v}^{I}(p) \gamma_{5} \gamma_{\mu} u_{v}^{J}(p)
$$

with $I, J$ being massive little group indices. Given the WNF for the heavy spinors, the WNF for the polarization vector can easily be computed by comparing Eq. (29) to its canonical polarization vector analog. We find that it is indeed given by Eq. (8c).

Applying Eq. (28) to Eq. (18) with the three-point KLT kernel $K_{3}=1$, we immediately recover the left-hand side of Eq. (24). For Compton scattering the KLT kernel is

$$
K_{4}=\frac{\left(s-m^{2}\right)\left(u-m^{2}\right)}{2 q_{1} \cdot q_{2}} .
$$

Then, applying Eq. (28) to the spin-1/2 HQET Compton amplitude with $k_{1}, q_{i} \cdot \epsilon_{j}, \epsilon_{i} \cdot \epsilon_{j} \neq 0$ up to and including terms of order $\mathcal{O}\left(\mathrm{m}^{-2}\right)$, we find Eq. (26) up to $\mathcal{O}\left(\mathrm{m}^{-1}\right)$. When imposing $k_{1}=q_{i} \cdot \epsilon_{j}=\epsilon_{i} \cdot \epsilon_{j}=0$, cancellations make the double copy valid up to $\mathcal{O}\left(\mathrm{m}^{-2}\right)$. The extension to higher inverse powers of the mass amounts to simply including the contributions of higher-order operators in the HQET and HBET amplitudes.

Therefore, by using Eq. (28) to convert heavy spinors in amplitudes to heavy polarization vectors, we have shown that 


$$
\left(\operatorname{HQET}_{s=1 / 2}\right) \times\left(\operatorname{HQET}_{s=1 / 2}\right)=\operatorname{HBET}_{s=1}
$$

for three-point and Compton amplitudes.

Conclusion.-We have shown that the three-point and Compton amplitudes derived from HQET can be double copied to those of HBET for spins $s \leq 1$. As long as the matter states of HQET and HBET are related through the double copy, in the sense described in the second section, and as long as higher-point amplitudes obey the spectral condition of Ref. [56], we see no obstacles to extending the double copy to higher-point amplitudes.

As mentioned in the introduction, due to the operator expansion of HPETs, the double-copy relation between HQET and HBET can be studied at each order in the $\hbar$ expansion, with the classical limit being of special interest. Studying the double copy of HPETs through this lens may provide some insight into the connection between the double copy with matter at the quantum and classical levels. We leave this study for future work.

This project has received funding from the European Union's Horizon 2020 research and innovation programme under the Marie Skłodowska-Curie grant agreement No. 764850 "SAGEX." The work of A. H. was supported in part by the Danish National Research Foundation (DNRF91) and the Carlsberg Foundation.

*kays.haddad@nbi.ku.dk

†ahelset@nbi.ku.dk

[1] H. Kawai, D. Lewellen, and S. Tye, Nucl. Phys. B269, 1 (1986).

[2] Z. Bern, J. J. M. Carrasco, and H. Johansson, Phys. Rev. D 78, 085011 (2008).

[3] Z. Bern, J. J. M. Carrasco, and H. Johansson, Phys. Rev. Lett. 105, 061602 (2010).

[4] Z. Bern, T. Dennen, Y.-t. Huang, and M. Kiermaier, Phys. Rev. D 82, 065003 (2010).

[5] Z. Bern, A. De Freitas, and H. L. Wong, Phys. Rev. Lett. 84, 3531 (2000).

[6] B. R. Holstein, Phys. Rev. D 74, 085002 (2006).

[7] J. Broedel and L. J. Dixon, J. High Energy Phys. 10 (2012) 091.

[8] N. Bjerrum-Bohr, J. F. Donoghue, and P. Vanhove, J. High Energy Phys. 02 (2014) 111.

[9] G. Chen and Y.-J. Du, J. High Energy Phys. 01 (2014) 061.

[10] H. Johansson and A. Ochirov, J. High Energy Phys. 11 (2015) 046.

[11] M. Chiodaroli, M. Günaydin, H. Johansson, and R. Roiban, J. High Energy Phys. 01 (2015) 081.

[12] F. Cachazo, S. He, and E. Y. Yuan, J. High Energy Phys. 07 (2015) 149.

[13] G. Chen, Y.-J. Du, S. Li, and H. Liu, J. High Energy Phys. 03 (2015) 156.

[14] Y.-J. Du and C.-H. Fu, J. High Energy Phys. 09 (2016) 174.

[15] M. Chiodaroli, M. Gunaydin, H. Johansson, and R. Roiban, J. High Energy Phys. 06 (2017) 064.
[16] E. Casali, Y. Geyer, L. Mason, R. Monteiro, and K. A. Roehrig, J. High Energy Phys. 11 (2015) 038.

[17] S. Stieberger and T. R. Taylor, Nucl. Phys. B913, 151 (2016).

[18] O. Schlotterer, J. High Energy Phys. 11 (2016) 074.

[19] D. Nandan, J. Plefka, O. Schlotterer, and C. Wen, J. High Energy Phys. 10 (2016) 070.

[20] C. Cheung, G. N. Remmen, C.-H. Shen, and C. Wen, J. High Energy Phys. 04 (2018) 129.

[21] E. Bergshoeff and P. Townsend, Nucl. Phys. B490, 145 (1997).

[22] E. Bergshoeff, R. Kallosh, T. Ortin, and G. Papadopoulos, Nucl. Phys. B502, 149 (1997).

[23] R. Kallosh, Lect. Notes Phys. 509, 49 (1998).

[24] E. Bergshoeff, F. Coomans, R. Kallosh, C. Shahbazi, and A. Van Proeyen, J. High Energy Phys. 08 (2013) 100.

[25] F. Cachazo, P. Cha, and S. Mizera, J. High Energy Phys. 06 (2016) 170.

[26] S. He and O. Schlotterer, Phys. Rev. Lett. 118, 161601 (2017).

[27] H. Elvang, M. Hadjiantonis, C. R. Jones, and S. Paranjape, J. High Energy Phys. 01 (2019) 195.

[28] C. Cheung and C.-H. Shen, Phys. Rev. Lett. 118, 121601 (2017).

[29] M. Chiodaroli, M. Gunaydin, H. Johansson, and R. Roiban, J. High Energy Phys. 07 (2017) 002.

[30] M. Chiodaroli, M. Gunaydin, H. Johansson, and R. Roiban, Phys. Rev. Lett. 120, 171601 (2018).

[31] F. Teng and B. Feng, J. High Energy Phys. 05 (2017) 075.

[32] Y.-J. Du, B. Feng, and F. Teng, J. High Energy Phys. 12 (2017) 038.

[33] C. Cheung, C.-H. Shen, and C. Wen, J. High Energy Phys. 02 (2018) 095.

[34] M. Chiodaroli, M. Günaydin, H. Johansson, and R. Roiban, J. High Energy Phys. 06 (2019) 099.

[35] A. Guevara, J. High Energy Phys. 04 (2019) 033.

[36] Z. Bern, A. Luna, R. Roiban, C.-H. Shen, and M. Zeng, arXiv:2005.03071.

[37] Z. Bern, J. J. Carrasco, M. Chiodaroli, H. Johansson, and R. Roiban, arXiv:1909.01358.

[38] H. Johansson and A. Ochirov, J. High Energy Phys. 01 (2016) 170.

[39] L. de la Cruz, A. Kniss, and S. Weinzierl, J. High Energy Phys. 09 (2015) 197.

[40] W. D. Goldberger and A. K. Ridgway, Phys. Rev. D 95, 125010 (2017).

[41] A. Luna, I. Nicholson, D. O'Connell, and C. D. White, J. High Energy Phys. 03 (2018) 044.

[42] R. W. Brown and S. G. Naculich, J. High Energy Phys. 03 (2018) 057.

[43] J. Plefka and W. Wormsbecher, Phys. Rev. D 98, 026011 (2018).

[44] J. Plefka, J. Steinhoff, and W. Wormsbecher, Phys. Rev. D 99, 024021 (2019).

[45] Z. Bern, C. Cheung, R. Roiban, C.-H. Shen, M. P. Solon, and M. Zeng, Phys. Rev. Lett. 122, 201603 (2019).

[46] Z. Bern, C. Cheung, R. Roiban, C.-H. Shen, M. P. Solon, and M. Zeng, J. High Energy Phys. 10 (2019) 206.

[47] Y. F. Bautista and A. Guevara, arXiv:1903.12419.

[48] J. Plefka, C. Shi, J. Steinhoff, and T. Wang, Phys. Rev. D 100, 086006 (2019).

[49] H. Johansson and A. Ochirov, J. High Energy Phys. 09 (2019) 040. 
[50] Y. F. Bautista and A. Guevara, arXiv:1908.11349.

[51] J. Plefka, C. Shi, and T. Wang, Phys. Rev. D 101, 066004 (2020).

[52] H. Georgi, Phys. Lett. B 240, 447 (1990).

[53] P. H. Damgaard, K. Haddad, and A. Helset, J. High Energy Phys. 11 (2019) 070.

[54] See Supplemental Material at http://link.aps.org/supplemental/ 10.1103/PhysRevLett.125.181603 for Lagrangians with heavy particles, which includes Ref. [55].
[55] C. D. Carone, Phys. Lett. B 253, 408 (1991).

[56] L. A. Johnson, C. R. Jones, and S. Paranjape, arXiv:2004.12948.

[57] H. Lehmann, K. Symanzik, and W. Zimmermann, Nuovo Cim. 1, 205 (1955).

[58] C.-H. Shen, J. High Energy Phys. 11 (2018) 162.

[59] R. Aoude, K. Haddad, and A. Helset, J. High Energy Phys. 05 (2020) 051. 